Visual Cognition, Volume 7, Issue 1 - 3 January 2000, pages 237 - 252

Psychology Press (Taylor \& Francis)

\title{
Limitations for Change Detection in Multiple Gabor Targets
}

Michael Wright, Alison Green and Stephen Baker

Department of Human Sciences, Brunel University, Uxbridge, Middlesex UB8 3PH, U.K.

\section{Abstract}

We investigate the limitations on the ability to detect when a target has changed, using Gabor targets as simple quantifiable stimuli. Using a partial report technique to equalise response variables, we show that the log of the Weber fraction for detecting a spatial frequency change is proportional to the log of the number of targets, with a set-size effect that is greater than that reported for visual search. This is not a simple perceptual limitation, because pre-cueing a single target out of four restores performance to the level found when only one target is present. It is argued that the primary limitation on performance is the division of attention across multiple targets, rather than decay within visual memory. However in a simplified change detection experiment without cueing, where only one target of the set changed, not only was the set size effect still larger, but it was greater at 2000 msec ISI than at 250 msec ISI, indicating a possible memory component. The steepness of the set size effects obtained suggests that even moderate complexity of a stimulus in terms of number of component objects can overload attentional processes, suggesting a possible low-level mechanism for change blindness.

\section{Introduction}

It is believed that a precise visual memory mediates the ability to discriminate between visual stimuli which are separated by a time interval. This has been called "sensory memory theory" (Lages \& Treisman, 1998). In contrast, the phenomenon of “change blindness” (Rensink, O’Regan \& Clark, 1997) might be defined as the inability to discriminate between stimuli which are separated by a time interval. To resolve the apparent contradiction in these two sets of results will require a careful analysis of the experimental conditions in which they arise.

In sensory memory experiments, Regan (1985) and Magnussen, Greenlee, Asplund \& Dyrnes (1990) used a briefly presented grating stimulus followed by an inter-stimulus interval (ISI) and then followed by another grating, and observers had to decide in which interval the higher spatial frequency grating appeared. Perhaps the most surprising result from these experiments was the apparent absence of decay. Magnussen \& Dyrnes, (1994), reported perfect storage for spatial frequency of gratings even over a 2 day period. However, others have found a gradual but statistically significant decay: Lee \& Harris (1996) found a gradual decay of memory for spatial contrast over a period of tens 
of seconds, Raney and Harvey (1996) found slight decay for spatial frequency discrimination, and Wright \& Gurney, (1995) found gradual decay for discrimination of orientation (a factor of two over $12.8 \mathrm{sec}$ ). Nevertheless, discrimination can be almost as good after an ISI of several seconds, as for a succcessive (zero ISI) or simultaneous discrimination, and this suggests that some elementary spatial and temporal attributes of visual images are represented in visual memory with almost the same order of precision with which they are perceived (Blake, Cepeda and Hiris, 1997).

In change blindness, however, the changes which remain undetected can be very large. Suprathreshold changes, such as the presence or absence of an object in a scene may remain undetected when the visual input is disrupted by a transient ISI, a saccade-like shift in position, or a transient mask. Usually, however, cueing the location of the changed object makes detection trivially easy. (O’Regan, Rensink and Clark, 1996; Simons, 1996; Rensink, O’Regan and Clark, 1997; Simons and Levin, 1997).

One important difference between the stimuli used in sensory memory and change blindness experiments is their complexity in terms of the number of component objects or targets. Most of the work on the psychophysics of visual memory has been conducted with single stimuli: for example, a single spatial frequency target is presented as $S_{A}$ again as $S_{B}$ after an ISI. However there have been a few studies using this experimental design with multiple stimuli (Raney and Harvey, 1995; Magnussen, Greenlee and Thomas, 1996; Baker and Wright, 1997, Greenlee and Magnussen, 1998). These studies show a strong deterioration in performance with increasing numbers of stimuli. Likewise, Luck \& Vogel (1997) presented a sample array, then an interstimulus interval, then a test array. The subjects' task was to indicate whether the two arrays were the same or different in terms of a single feature. They found that performance was accurate, for coloured squares, or for oriented bars, for arrays up to four items, and deteriorated thereafter.

This limited capacity of visual memory may conceivably contribute to the phenomenon of change blindness. However in sensory memory tasks, detecting change amongst arrays of objects, there are possible limitations on performance other than memory. Specifically, there are reasons to suppose that both perceptual analysis and the allocation of attentional resources are affected by the number of targets present. These other sources need to be identified and explored before any strong conclusions about memory capacity limitations are drawn. Palmer (1995) has shown that increasing set size leads to decrease in performance in a wide variety of visual tasks involving selection of a target, and that these set size effects could be due to perceptual limitation, limitations on selection, limitations on processing the selected object, or limitations on decision processes. All these possibilities are additional to possible limitations in memory storage capacity.

A possible function that has been proposed for visual memory is integration of retinotopic information across saccades to form a stable three-dimensional 
representation of the spatial layout of the environment (Irwin, 1992). Integration across saccades does at least preserve simple positional information (Hayhoe, Lachter \& Feldman, 1991). Work on change blindness, however, indicates that the amount of image information retained across saccades or transient image changes is severely limited. If visual memory did have a high capacity, the radical changes in visual appearances which occur between saccades might overload the system (just as the memory capacity of present-day computers is taxed by highresolution, real-time, continuous image acquisition). Human observers are insensitive to large changes in a complex scene especially when these changes coincide with a shift in the image requiring a saccade, (Blackmore, Brelstaff, Nelson \& Troscianko, 1995) or a blank interstimulus interval (Rensink, O’Regan \& Clark, 1997).

There are similarities and differences between the experimental procedures used in change blindness experiments and sensory memory experiments. Typically in both paradigms, two stimuli $\mathrm{S}_{\mathrm{A}}$ and $\mathrm{S}_{\mathrm{B}}$ are presented (separated by a time interval, ISI), and the observer's task is to discriminate the two stimuli. In sensory memory experiments, psychophysical thresholds are often measured, whereas in change blindness experiments, the critical changes are generally measured with a reaction time or "percent detected" measure.

A psychophysical approach to change blindness, using simple stimuli, has been developed by Orbach and Scott-Brown $(1997,1998)$, Scott-Brown and Orbach (1998) and Scott-Brown, Baker and Orbach (1999). Thus, Scott-Brown and Orbach (1998) showed a striking increase in contrast increment thresholds in stimuli consisting of five mixed-contrast elements. Large contrast changes which could not be detected in the multiple element displays were easily detectable in single-element displays, or when the changed element was cued, implying an involvement of low-level visual mechanisms in change blindness.

The aim of the present experiments likewise is to identify possible low-level visual processes which could contribute to the phenomenon of change blindness. The approach adopted will be to use a sensory memory experimental design, in which sets of stimuli $S_{A}$ and $S_{B}$ are presented separated by an ISI. The task will be to detect a change in spatial frequency between $S_{A}$ and $S_{B}$ as a function of the number of targets. The data will be analysed in relation to previous data on set size effects, and the possible locus of obtained set size effects will be analysed by varying the type of stimulus cueing and the ISI. The results will be interpreted in terms of the possible contributions of perceptual, attentional, memory and decision processes in the detection of stimulus change.

\section{Methods}

\section{Apparatus}

Stimuli were generated using a VSG (Visual Stimulus Generator ) which is a specialized graphics card made by Cambridge Research Systems, U.K., installed in a DAN 468 computer. The VSG card was controlled by a program written in Pascal. Stimuli were presented on an Eizo T662T flexiscan display monitor using a frame rate of $100 \mathrm{~Hz}$. 


\section{Stimuli}

The stimuli used were circular Gabor patches which are made by multiplying a sine-wave luminance grating with a circular Gaussian function. The standard deviation of the Gaussian was $0.47 \mathrm{deg}$. The mean Spatial frequency of the gratings was $4 \mathrm{c} / \mathrm{deg}$. The Gabor patches were presented in two successively presented arrays, $\mathrm{S}_{\mathrm{A}}$ and $\mathrm{S}_{\mathrm{B}}$, each containing one, two, three or four patches. The centre of each patch was 1 deg from the centre of the fixation cross. For details of the geometry and timing of the stimulus, see Figure 1.

Fig.1. Spatial and temporal arrangement of stimuli in a single trial (4 Gabor, 2000 msec condition).

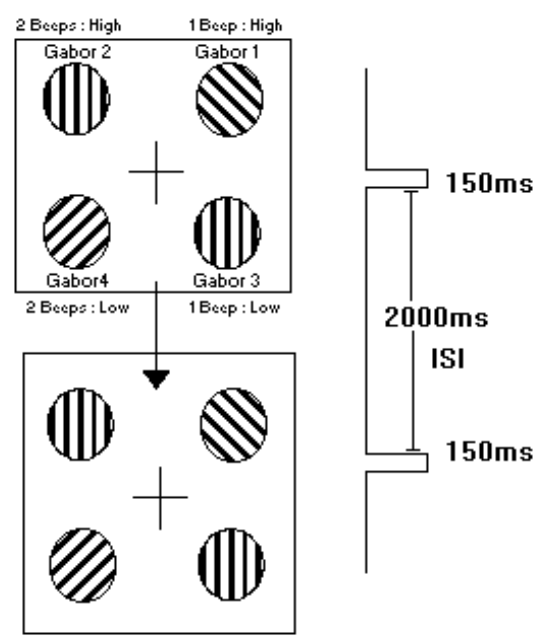

Between trials, the Orientation of the gratings was randomized between vertical, horizontal and 45 degree diagonal orientations, but the orientations of patches in corresponding positions of $S_{A}$ and $S_{B}$ was always the same within a trial. The reason for this randomization was to prevent the build-up of gestalts for the whole pattern. The Phase of bars in a grating is their relative position within the Gaussian envelope of the Gabor patch. In all of the experiments phase was randomized, between $S_{A}$ and $S_{B}$ so as to eliminate the automatic detection of changes by apparent motion cues. Contrast is defined as the difference of the maximum and minimum luminance values divided by their sum (Michelson contrast) and is expressed as a percentage. Contrast was kept at the maximum (95\%) with all stimuli.The stimuli and their background had a mean luminance of $40 \mathrm{~cd} / \mathrm{m}^{2}$ and were viewed at 2.65 metres in an enclosure with a constant luminance around the monitor of $5 \mathrm{~cd} / \mathrm{m}^{2}$.

Figure 1 shows the stimulus presentation conditions for the experiment (2000 msec ISI condition). On each trial, the appearance of the fixation cross ( $0.25 \mathrm{deg})$ preceded the first stimulus by $800 \mathrm{msec}$, and the cross remained on until the response button was pressed. The first set of Gabors was displayed for $150 \mathrm{msec}$, which was followed by an inter-stimulus interval during which the screen was 
blanked to mean luminance, which was then followed by the second set of Gabors, again displayed for 150 msec.

\section{Design}

The main independent variables were a). The number of Gabor targets $(1,2,3$ or 4), b). The inter-stimulus interval $(0,250$, or $2000 \mathrm{msec}), \mathrm{c})$ the cueing condition (pre-cued, post-cued or uncued, visual or auditory cue). The dependent variable was the spatial frequency difference between the target in $\mathrm{S}_{\mathrm{A}}$ and the corresponding target $S_{B}$ required to detect a change on $75 \%$ of trials.

\section{Procedure.}

The observer responded with one of two buttons. One button was for indicating a perceived change in the spatial frequency, the other that no change was noticed. The 9 equally-spaced constant stimuli represented spatial frequency changes of between -0.8 and $+0.8 \mathrm{c} / \mathrm{deg}$ in steps of $0.2 \mathrm{c} / \mathrm{deg}$. On $50 \%$ of the trials the spatial frequency stayed the same (i.e. middle increment $=0$ ) and on the other $50 \%$ it changed. So after one run (96 trials) the middle stimulus value of zero change had been used $50 \%$ of the time (48 trials in all) and each of the changing constant stimuli were randomly used 6 times. Each run was repeated 4 times. Each data point in every graph in this paper is based on a complete (double) psychometric function, and is thus based upon 4x96 = 384 single trials.

The Gabor patch to be discriminated had a randomized initial spatial frequency which meant both $S_{A}$ and $S_{B}$ were constrained to a value between 3.2 and 4.8 $\mathrm{c} / \mathrm{deg}$. The reason for the randomisation was to prevent the discrimination becoming soluble by identification of absolute spatial frequency either in interval $\mathrm{S}_{\mathrm{A}}$ or in $\mathrm{S}_{\mathrm{B}}$. The position of the cued Gabor was randomized between the four possible positions.

In Experiment 1 and 2, each of the uncued Gabors was also randomized in a similar way to the cued Gabor, each initially starting with a randomized spatial frequency between 3.2 and $4.8 \mathrm{c} / \mathrm{deg}$, and then changing on $50 \%$ trials and remaining unchanged on the other $50 \%$.

In Experiment 3, there was no cueing, and the non-target Gabors did not change. The target Gabor changed, as before, on 50\% of trials. The task in Experiment 3 was thus to determine whether a spatial frequency change had occurred anywhere, in any of the targets, or not.

\section{Subjects}

The observers in Experiment 1, 2 and 3 were MW and EG. Additionally DW participated in Experiment 3. All had normal acuity and contrast sensitivity. MW was an author of this paper, and EG and DW were unaware of the experimental hypotheses, and were paid $£ 5$ per hour for their participation.

\section{Data analysis}


For each experimental condition, the probability of detecting change was plotted against the magnitude of the change. This generated a psychometric function with two approximately symmetrical limbs, one for spatial frequency increments and one for decrements. Separate curves were then fitted to each limb of the psychometric function, and from each of these fitted curves, a $75 \%$ correct detection threshold was determined. Since there were no systematic differences in the detection of increments and decrements, these two thresholds (ignoring sign) were combined to give a mean and standard error of the change detection threshold. This specified by how much the spatial frequency of the cued target stimulus had to change for it to be detected reliably better than chance. Since the task was a discrimination task, the threshold could be expressed as a Weber fraction, that is the change detection threshold (in c/deg) divided by the mean spatial frequency $(4 \mathrm{c} / \mathrm{deg})$ of the target.

\section{Results.}

\section{Experiment 1.}

The purpose of the first experiment was to determine whether perceptual factors were significant in determining the dependence of change detection on numbers of stimuli. There were 16 different conditions in all; a single-target condition, plus a 4 target post-cued condition, plus two 4 target pre-cued conditions. Each of these condition was repeated at ISI's of 0, 250 and $2000 \mathrm{msec}$.

Under the post-cued (partial report) condition, an auditory tone (high-low, singledouble) immediately following $S_{B}$ indicated to the subject the position of the Gabor stimulus (i.e. top left, top right, bottom left or bottom right) which was to be reported. In the corresponding pre-cued condition, the tone was presented 400 msec before $S_{A}$, and this allowed subjects to pre-select which of the four successive Gabor-pairs should be compared. Subjects voluntarily maintained fixation and did not look directly at the indicated target, but to check the effectiveness of this voluntary effort, a control condition was introduced. In the control condition, a small dot appeared in one corner of the fixation cross. The quadrant in which the dot appeared corresponded with the selected target stimulus. It appeared immediately before $S_{A}$ and was present for $20 \mathrm{msec}$, so there was insufficient time to make an anticipatory saccade to the target. It was necessary to maintain central fixation in order to see this pre-cue.

The effect of a pre-cue was the same whether it was a visual cue or an auditory cue. Since the visual cue condition controlled for possible changes in fixation consequent upon the cue, this result suggests that any possible eye movements in the auditory precue condition did not seriously affect discrimination.

In the following comparison, the Weber fraction for spatial frequency change in a single target is compared with the Weber fraction when four targets are present.

Fig.2. Weber fractions for detecting spatial frequency change. a) subject EG $b$ ) subject MW. The ISI's used were 0, 250 and 2000 msec. The first group of bars shows data for a single patch. The second group of bars shows worse discrimination performance for four Gabor patches, where the subject had to report only on a target patch indicated by an auditory post-cue. The third group 
of bars shows that pre-cueing the target patch in an array of four patches gives equivalent performance to a single patch. The fourth group of bars shows that a visual pre-cue visible at the fixation cross has the same effect as an auditory precue.

Data from 'PEXP.CGD'

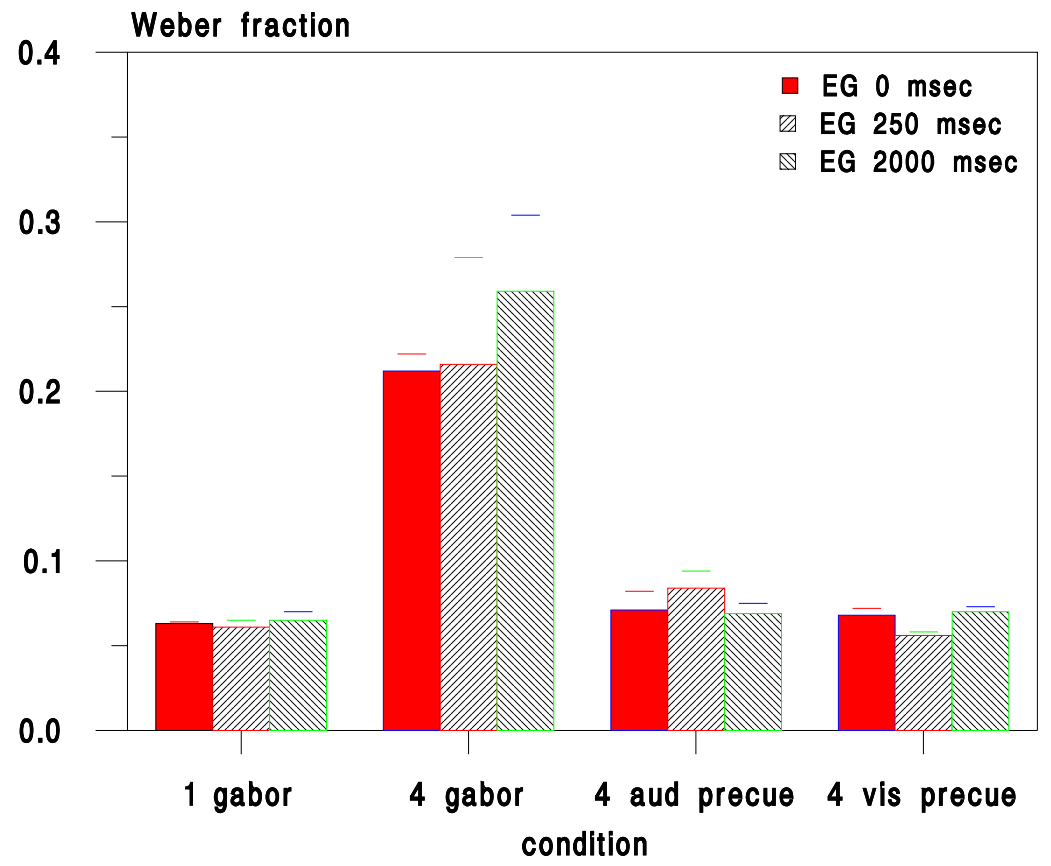

Data from 'PEXP.CGD'

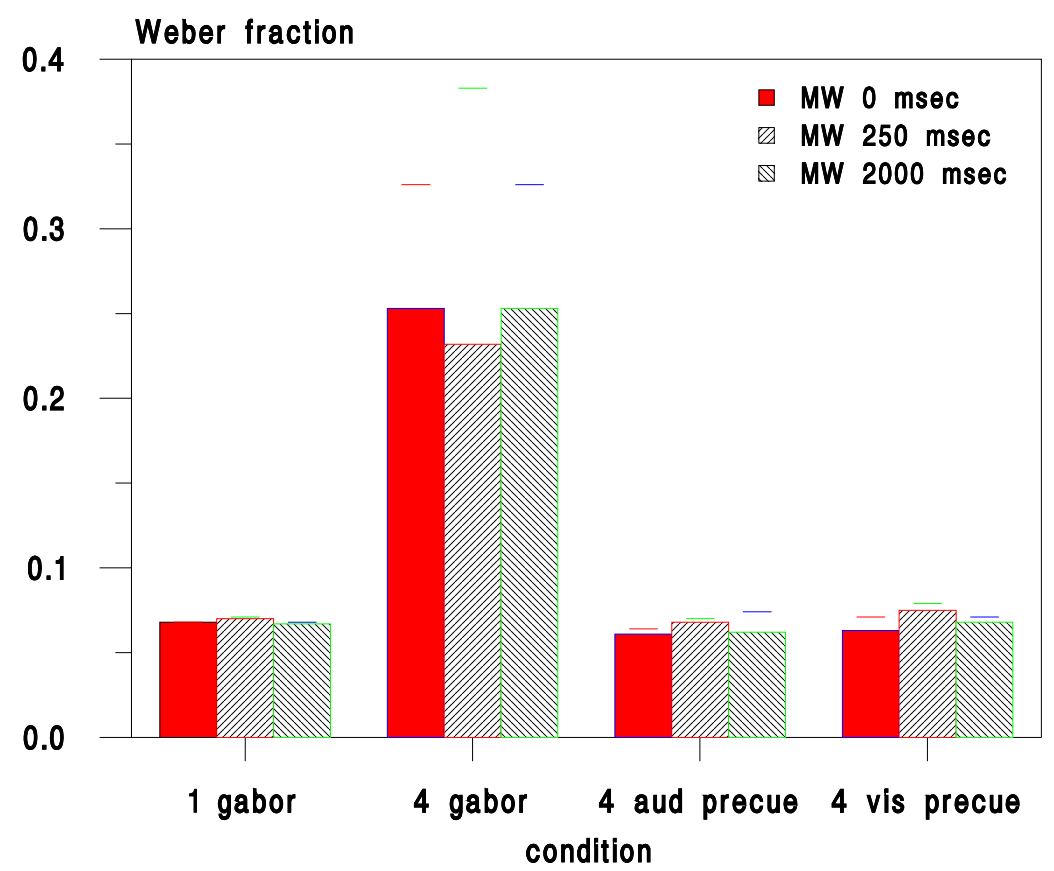

Looking at the first two groups of bars, it can be seen that Weber fractions for spatial frequency change are much smaller for a single target than for four targets. 
Since a partial report technique was used for the four-target condition, the worse performance cannot be due to reponse competition. Also, the effect of a pre-cue is the same whether it is a visual cue or an auditory cue (third and fourth groups of bars). This is good indirect evidence that fixation control in the experiments was satisfactory, and therefore that the comparisons across conditions are not confounded by variations in visual field location. Thus, when a pre-cue is given, allowing subjects to select the target stimulus as soon as it is presented, performance is as good as it is with a single target (third group of bars). We will argue from this that the limitation on performance in the post-cued four-target condition is not due to stimulus crowding effects or effects of parafoveal viewing, since the four Gabor targets are exactly equivalent in the pre-cued cases. The limitation is therefore not primarily a perceptual one.

\section{Experiment 2.}

Next, we shall turn to the effect of the number of targets, in an attempt to discover whether there is a set-size effect in the detection of stimulus change. In addition to the one-target and four-target post-cued conditions in experiment 1, further data were obtained for two and three Gabor targets. The geometry of the stimulus was identical in Experiments 1 and 2.

Following the method of Palmer (1994), we first plotted Weber fractions for spatial frequency change detection against the number of Gabor targets, on loglog co-ordinates. Weber fractions increased with stimulus set size.

We found that thresholds for stimulus change increase as the number of targets increases. The results are shown in Figure 3.

Fig.3. Set-size effects for detecting spatial frequency change in arrays of Gabor patches. The target patch is cued by an auditory signal after the second presentation $S_{B}$. The ordinate shows the log of the Weber fraction (75\% detection threshold for spatial frequency discrimination divided by mean base spatial frequency). The abscissa shows the log of the number of patches. All discriminations were post-cued, so that only a single patch position had to be reported on. Data for $0 \mathrm{msec}, 250 \mathrm{msec}$ and $2000 \mathrm{msec}$ ISI are shown. a) Subject $E G$ b) subject $M W$ 
Data from 'GN3EXP.CGD'

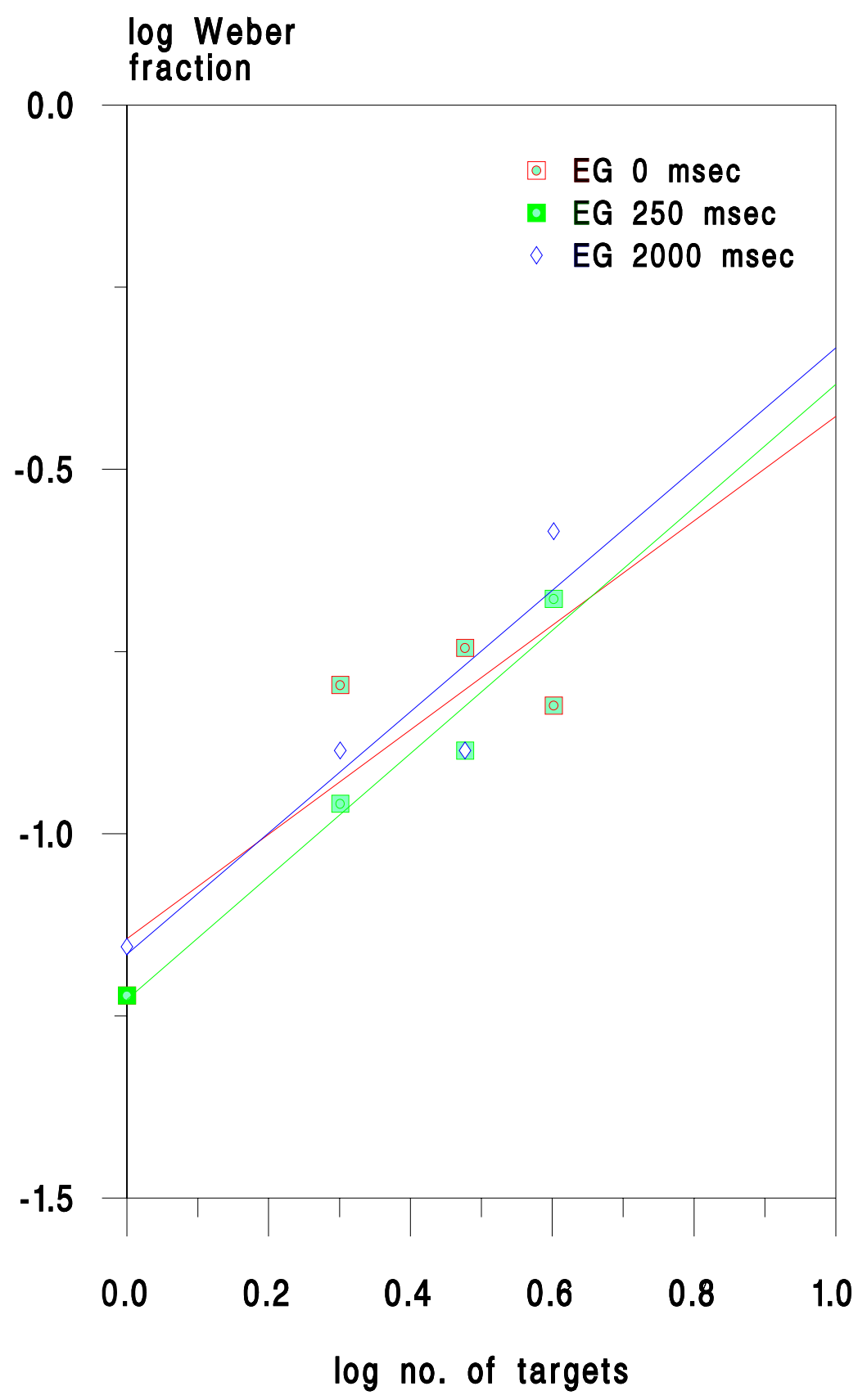




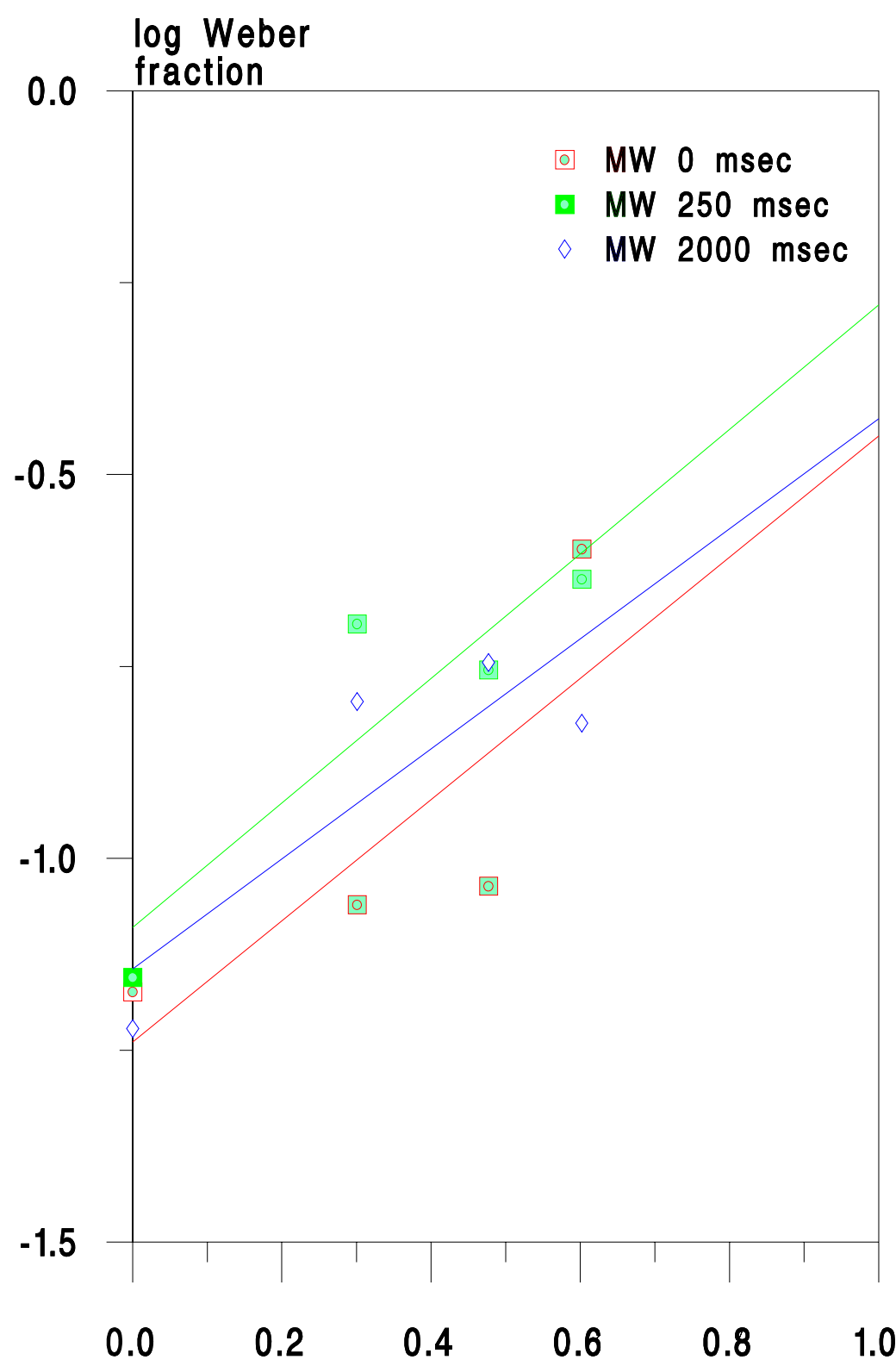

$\log$ no. of targets

On the ordinate we show the log of the Weber fraction for spatial frequency change, and on the abscissa we show the log of the number of targets. Results for both subjects show that discrimination decreases with the log of the number of targets, and a similar rate of decline is seen for 0, 250 and $2000 \mathrm{msec}$ ISI. Note that in all conditions, including the 0 msec condition, a randomized phase change was always present in all stimuli, so that the change in spatial frequency could not merely be detected by the apparent movement it produces. A straight line may be fitted to the data and the slopes are similar for all conditions. For subject EG, the slopes obtained were: $0 \mathrm{msec}=0.72\left(\mathrm{r}^{2}=0.73\right), 250 \mathrm{msec}=0.84\left(\mathrm{r}^{2}=0.96\right), 2000$ 
msec $=0.83\left(r^{2}=0.87\right)$. For subject MW, the slopes obtained were: $0 \mathrm{msec}=0.79$ $\left(r^{2}=0.66\right), 250 \mathrm{msec}=0.81\left(\mathrm{r}^{2}=0.81\right), 2000 \mathrm{msec}=0.72\left(\mathrm{r}^{2}=0.73\right)$.

On this basis it would appear that the main determinant of spatial frequency discrimination thresholds is set size, and there is no difference in performance due to ISI. However the steepness (or magnitude) of the set-size effect is greater than that found by Palmer for visual search tasks. This will be considered in the Discusssion.

\section{Experiment 3.}

It might be argued that experiments 1 and 2 differ somewhat from a typical change blindness experiment, in that the non-target stimuli are capable of changing on any trial as well as the target stimulus, and in the use of post-cueing. Thus, the experiments could be made more similar to typical change blindness experiments, by removing the cue, and by limiting the change to a single stimulus object. This was done in Experiment 3. On any one trial, one target changed in spatial frequency with 50\% probability, whereas the remaining targets changed with $0 \%$ probability. For multiple targets, the location of the changeable target was randomized from trial to trial. However, the subject did not have to identify the location of the change, merely to indicate whether change had occurred anywhere in the stimulus. As before, all targets had a random phase change to prevent a trivial solution by the detection of apparent motion. The spatial frequency of $\mathrm{S}_{\mathrm{A}}$ Gabors was randomized as before to eliminate cues from absolute spatial frequency values. The results are shown in Figure 4.

Fig.4. Set-size effects for detecting spatial frequency change in arrays of Gabor patches. The target patch is uncued, but only one patch can change on any trial. The subject's decision is thus to indicate whether anything has changed in the array of one to four patches, comparing between $S_{A}$ and $S_{B}$. The ordinate shows the log of the Weber fraction (75\% detection threshold for spatial frequency discrimination divided by mean base spatial frequency). Data for $250 \mathrm{msec}$ and 2000 msec ISI are shown. a) Subject EG b) subject MW (two sets of data) c) subject $D W$. 
Data from 'NOCUEDE.CGD'

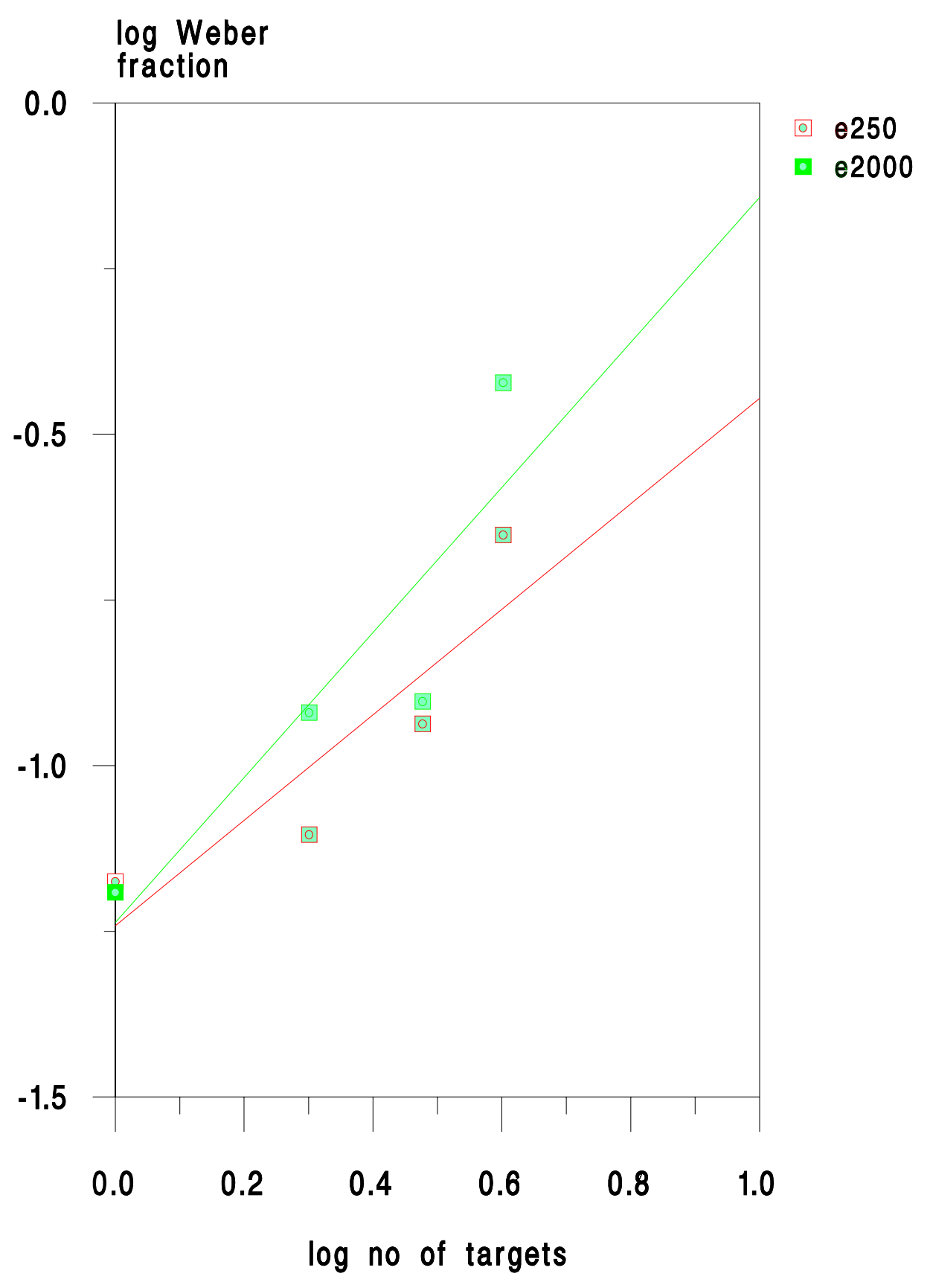


Visual Cognition, Volume 7, Issue 1 - 3 January 2000, pages 237 - 252

Psychology Press (Taylor \& Francis)

Data from 'NO3CUE.CGD'

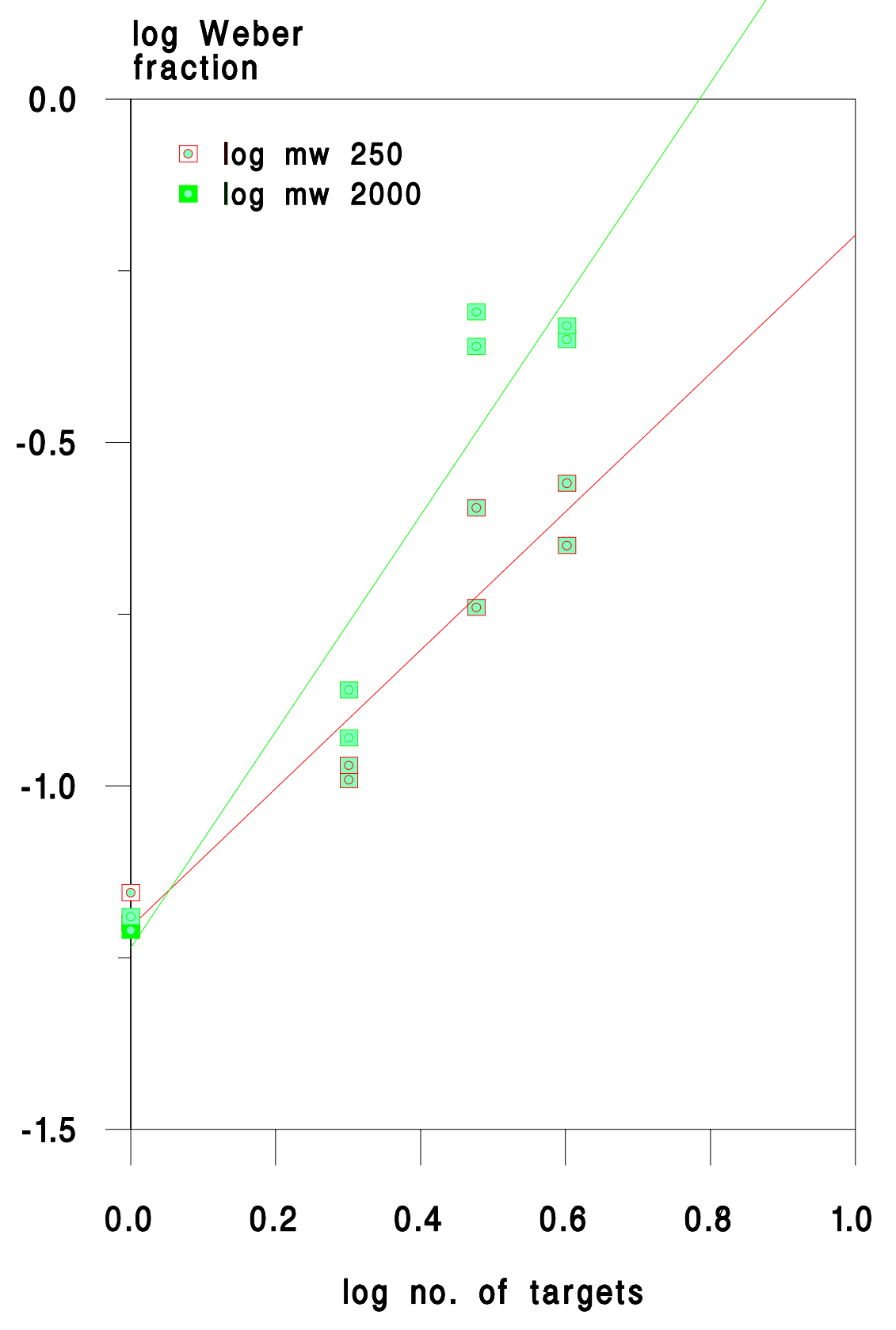


Visual Cognition, Volume 7, Issue 1 - 3 January 2000, pages 237 - 252 Psychology Press (Taylor \& Francis)

Data from 'NOCUEDE.CGD'

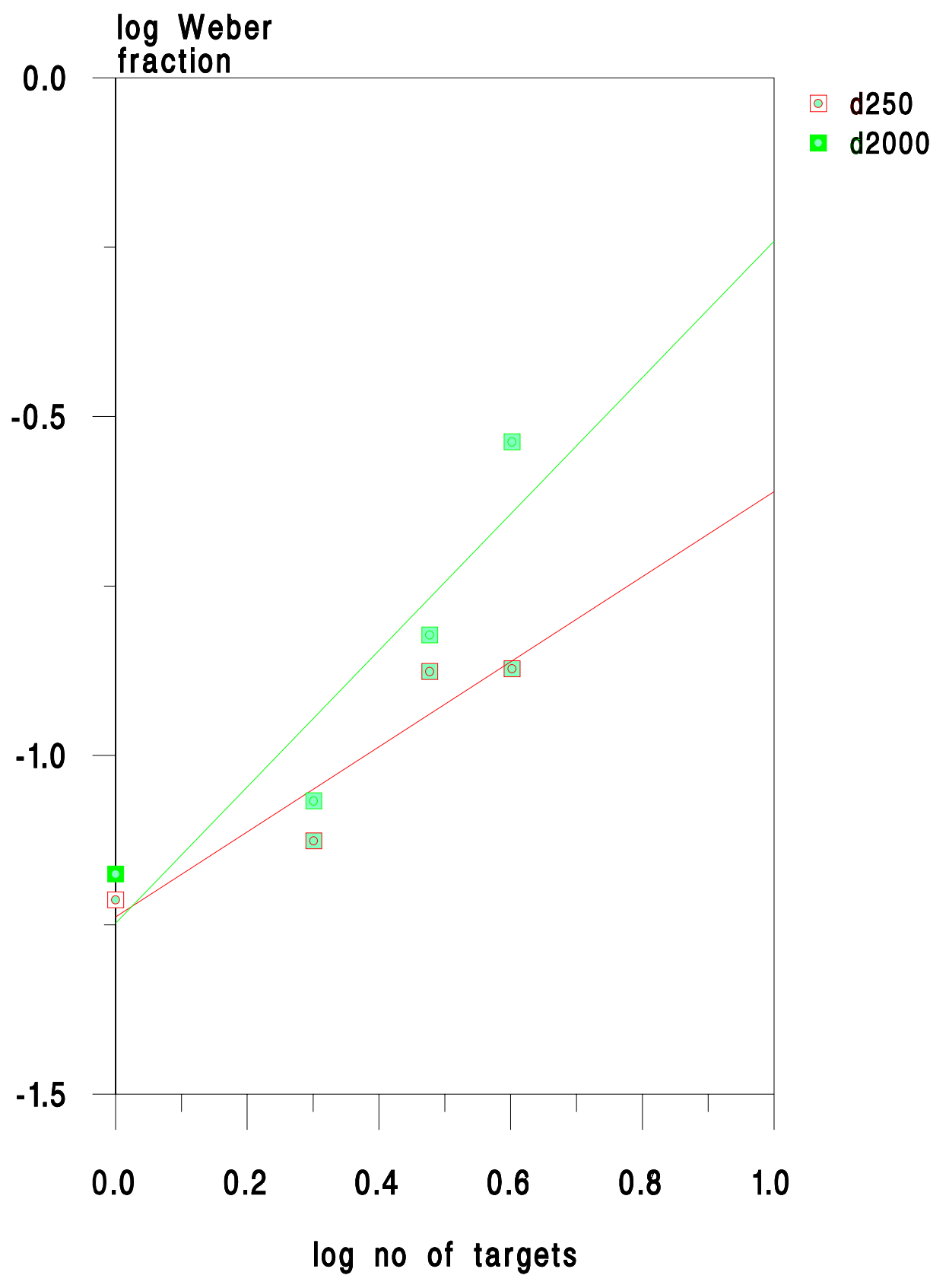

For subject EG, the slopes obtained were: $250 \mathrm{msec}=0.80\left(\mathrm{r}^{2}=0.99\right), 2000 \mathrm{msec}$ $=1.09\left(\mathrm{r}^{2}=0.94\right)$. For subject MW, the slopes obtained were: $250 \mathrm{msec}=1.01$ $\left(r^{2}=0.92\right), 2000 \mathrm{msec}=1.57\left(r^{2}=0.92\right)$. For subject DW the slopes obtained were : $250 \mathrm{msec}=0.63\left(\mathrm{r}^{2}=0.92\right), 2000 \mathrm{msec}=1.01\left(\mathrm{r}^{2}=0.92\right)$.

Once again there is a strong set-size effect. The slopes (at $2000 \mathrm{msec}$ ) are steeper in the no-cue experiment than in the previous experiment with cueing. Moreover, 
there is in this experiment an effect of ISI duration, which did not appear in experiment 2. The magnitude of the set-size effect is greater with a $2000 \mathrm{msec}$ ISI than with a 250 msec ISI.

\section{Discussion}

The results indicate that spatial frequency changes which are easily detectable in a single target become undetectable when the number of targets is increased. They implicate the involvement of low-level visual processes in the phenomenon of change blindness. Our independent results and interpretations thus support those of Scott-Brown and Orbach (1998) in a prior study.

The effect of cueing in restoring detectability of change, which is characteristic of change blindness, was seen in Experiment 1. To be effective, the cueing must precede rather than follow the stimuli. The ability to detect change in multiple targets was (in experiment 2) independent of ISI. Thus the results presented here are entirely consistent with the sensory memory literature which suggests that the accuracy of visual discriminations is as good after a short delay as for temporally contiguous presentation, that is, that sensory memory has a high fidelity and persistence although decay has been reported in some sensory memory experiments.

The limitation due to the number of targets in the display was the same for 0,250 and 2000 msec ISI. However, in experiment 3, there was a clear effect of ISI, with larger set-size effects occuring with $2000 \mathrm{msec}$ ISI than at $250 \mathrm{msec}$ ISI. From the graphs relating Weber fractions for detecting spatial frequency change to the number of Gabor targets, it is evident that there is a general increase in discrimination threshold with the increasing number of Gabors.

We can rule out a simple perceptual explanation of these results. In Experiment 1, the 4 target stimulus was identical in the pre-cued and post-cued condition.

However in the pre-cued condition there was no set size effect: the discrimination threshold was identical for the one-target and four-target stimulus. This suggests that there is no degradation of performance due to a reduction in discriminability of multiple stimuli (deHaan, Lutz \& Noest, 1996).

Could the set size effect be due to response factors? The post-cueing conditions use a variety of "partial report” (Sperling, 1960). Although up to four stimuli have to be remembered from the first presentation, to carry out the discrimination, only one comparison needs to be made with the second set of stimuli, and only one response needs to be given.. Therefore the observed decrement in performance with set size is not due to response competition.

Could the limiting factor be memory? It is not compelling that memory decay contributes to the set size effect seen in Experiment 2, since the effect was the same for $0 \mathrm{sec}$ ISI as for $250 \mathrm{msec}$ or $1 \mathrm{sec}$ ISI. Orbach and Scott-Brown (1998) 
and Scott-Brown, Baker and Orbach (1999) also considered that factors other than memory were primary, because the same threshold increases were found for spatial as for temporal comparison of multiple targets. However, in our experiment 3 , there was an additional effect due to the ISI since a different magnitude (slope) of the set size effect was found in the $2000 \mathrm{msec}$ and $250 \mathrm{msec}$ conditions. This suggests that the effect of multiple stimuli is stronger at longer ISI in the non-cued condition, whereas the effect of ISI delay is negligible in the post-cued condition.

Set-size effects, in which thresholds increase with increasing number of elements, have been found in a wide range of visual tasks (Palmer, Ames and Lindsey, 1993). Thus, Palmer et al. (1993) found a slope of 0.25 for a line length discrimination task, Palmer (1994) found the same value for discrimination of contrast, luminance, orientation, size and colour. This value of 0.25 was consistent , for yes-no tasks, with the predictions of a particular model, the decision integration hypothesis. This is an extension of signal detection theory to explain the integration of information from multiple stimuli (Green and Swets, 1988). Each additional stimulus contributes an additional source of noisy information, and thus an additional opportunity for false positive responses, and it is this effect which leads to a deterioration of performance with multiple stimuli. The decision integration hypothesis predicts the same set-size effect independent of the stimulus, once effects of stimulus coding are taken into account. Palmer (1994) argued that the effect of integrating a decision across multiple stimuli was attentional, because the subject could decide which stimuli to monitor.

However, in contrast with the effects reported by Palmer $(1994,1995)$, the slopes of the set-size effects reported in the present study are much greater. Thus in experiment 2, the mean slope of the set-size effect (averaged across subjects and ISI conditions) was 0.79 . In experiment 3, it was 1.01 for the $250 \mathrm{msec}$ condition, and 1.57 for the 2000 msec condition. Thus the conclusion seems inescapable that the set size effect reported here is considerably larger than that reported by Palmer (1994). A simple check on the figures confirms this: for example, spatial frequency difference thresholds for MW's spatial frequency discrimination are $0.25 \mathrm{c} / \mathrm{deg}$ for a single target and $1.03 \mathrm{c} / \mathrm{deg}$ for four targets. With a mean base spatial frequency of $4 \mathrm{c} / \mathrm{deg}$ this gives Weber fractions of $0.0625(\log =-1.2)$ and 0.26 (log=-0.59) respectively. Clearly these effects are much stronger than those seen in visual search tasks.

The second hypothesis considered by Palmer (1994) was capacity-limited attention hypothesis. On this view, the perceptual system has a limited capacity, such that with multiple stimuli, limited processing resources are distributed across all the stimuli, so that the information that it is possible to extract from a single stimulus is inversely proportional to the number of stimuli. This predicts slopes for the set-size effect of the order of 0.75. Thus Palmer's (1994) results were not consistent with the perceptual coding hypothesis. However, one of the results in the present study (Experiment 2) is in the general range of this prediction, and the results of Experiment 3 are somewhat greater. 
The slopes of the set-size effects obtained in the present study are consistent with the value of 0.98 obtained by Scott-Brown and Orbach (1998) for contrast increment thresholds in multiple mixed contrast element displays. It is relevant that in the present study, as in that of Scott-Brown and Orbach (1998), non-target elements were non-uniform, whereas the visual search tasks employed by Palmer (1994) utilised uniform distractor elements. Scott-Brown and Orbach (1998) moreover, found that the slope of the set-size effect for uniform contrast displays was consistent with the 0.25 predicted by the decision integration hypothesis. Thus, the capacity-limited attention hypothesis cannot account for both the uniform and non-uniform distractor tasks, although it is possibly consistent with the non-uniform distractor results, including the present results.

There are several possible differences in experimental conditions between the present experiments and the set-size experiments of Palmer (1994) that could explain the different results. Most of Palmer's examples are simple visual search tasks in which the target stimulus must be discriminated from a number of distractors in a single stimulus presentation. In the present experiments, the target is not identifiable in a single presentation. It is only by comparing corresponding elements in the first and second exposures that the discrimination can be solved. Thus, instead of each element acting as a distractor, and thus changing the signal and noise distributions in a single decision process, there is a parallel process in which all target pairs need to be processed. This is equivalent to carrying out multiple discriminations in parallel.

A similar example of interaction between parallel decision processes is given by Greenlee and Magnussen (1998), who found that when subjects discriminated two stimulus targets with respect to spatial frequency, thresholds were raised 3-6 times compared with single discriminations. They suggest that there is interference between stimuli when they need to be processed along a single dimension. This hypothesis is equivalent to the perceptual coding hypothesis described by Palmer (1994), but suggests that the limitation on perceptual coding is apparent only for discriminations involving a single dimension of perceptual encoding (such as spatial frequency). It is evident also that it not a simple perceptual input effect like crowding, but concerns the allocation of attentional resources to multiple discriminations.

Finally, the results of Experiment 3 produced the largest set-size effects of all in the 2000 msec condition. This is despite the fact that the task seemed to require less cognitive effort than that of experiment 2, in that it was not necessary to think about the consequences of a cue signal. This experiment was the most similar to the change blindness experiments, in that only a single stimulus element could change on a given trial. However, the task did require the parallel processing of all stimuli. The fact that there was an effect of ISI in this task, but not in the postcued task, suggests that it is there is an aspect of the task that shows memory 
decay. Thus it is unlikely that a parallel allocation of attentional resources can account for all the findings reported.

The relevance of these findings to change blindness phenomena needs to be explored further. However, it is possible to draw some tentative conclusions. Firstly, there are strong effects of stimulus complexity on the ability to detect change. Change blindness typically occurs in stimulus displays containing moderate to high numbers of objects (though this is somewhat arbitrary to quantify). What we show here are strong set-size effects which are apparent even in arrays of four or fewer simple stimuli: and these set size effects can be much greater in a change-detection experiment than in a visual search experiment.

One difference between the present experiments and change blindness experiments is the use of Weber fractions as a dependent measure. The huge suprathreshold changes that subjects (in change blindness experiments) fail to detect are simply not quantifiable in this way. However extrapolation of the set size effect in multiple Gabor stimuli seen here indicates that Weber fractions would become for practical purposes unmeasurably large given moderate increases in stimulus number.

The experiments reported here suggest that change blindness phenomena are in part due to limited-capacity low level mechanisms of visual attention which can be studied quantitatively using established psychophysical methods. In particular, to detect change in successively presented scenes would seem to require parallel deployment of attentional resources over multiple elements in those scenes.

Acknowledgments: We thank Frank Durgin and Harry Orbach for their detailed and useful comments.

\section{References}

Baker, S. and Wright, M.J. (1997) Visual memory for multiple Gabor targets. Perception, 26, Suppl. 125a.

Blake, R., Cepeda, N.J. \& Hiris, E. (1997). Memory for visual motion. Journal of Experimental Psychology: Human Perception and performance, 23, 353-369.

Blackmore, S.J, Brelstaff, G., Nelson, K, \& Troscianko, T. (1995) Is the richness of our visual world an illusion? Transsaccadic memory for complex scenes.

Perception, 24, 1075-1082.

Dehaan, E., Lutz, C. \& Noest , A. J. (1996) Nonspatial visual-attention explained by spatial attention plus limited storage. Perception, 25, 591-608 
Green, D. M. \& Swets, J. A. (1988). Signal detection theory and psychophysics Peninsula.

Greenlee, M.W. \& Magnussen, S. (1998). Limited-capacity mechanisms of visual discrimination. Vision Research, 38, 375-385.

Hayhoe, M., Lachter, J. \& Feldman, J. (1991). Integration of form across saccadic eye movements. Perception, 20, 393-402.

Irwin, D. E. (1992). Memory for position and identity across eye movements. Journal of Experimental Psychology: Learning, Memory, and Cognition, 18 307317

Lages, M. \& Treisman, M. (1998). Spatial frequency discrimination: visual long term memory or criterion setting? Vision Research, 38, 557-572.

Lee, B. \& Harris, J. (1996). Contrast transfer characteristics of visual short term memory. Vision Research, 36, 2159-2166.

Luck, S.J. \& Vogel, E.K. (1997). The capacity of visual working memory for features and conjunctions. Nature, .390, 279-281.

Magnussen, S. \& Dyrnes, S. (1994). High-fidelity perceptual long-term memory. Psychological Science, 5, 99-102.

Magnussen, S. Greenlee, M. W. (1993). Visual short-term memory for spatial frequency components presented concurrently or sequentially. Investigative Ophthalmology and Visual Science, 34, 782-782.

Magnussen. S., Greenlee, M. W., Asplund, R. \& Dyrnes, S. (1991). Stimulusspecific mechanisms of visual short-term memory. Vision Research, 31, 12131219.

Magnussen, S., Greenlee, M. W., Asplund, R. \& Dyrnes, S. (1990). Perfect visual short-term memory for periodic patterns. European Journal of Cognitive Psychology, 2, 345-362.

Magnussen, S., Greenlee, M. W. \& Thomas, J. P. (1996). Parallel processing in visual short-term memory. Journal of Experimental Psychology: Human Perception and Performance, 22, 202-212. 
Orbach, H.S. and Scott-Brown, K.C. (1997). "Change blindness” and contrast discrimination thresholds. Investigative Ophthalmology and Visual Science (Suppl.), 38, 1.

Orbach, H.S. and Scott-Brown, K.C. (1997). Change blindness is comparison blindness. Investigative Ophthalmology and Visual Science (Suppl.), 38, 1.

O’Regan, J.K., Rensink, R.A. and Clark, J.J. (1996). “Mud splashes” render picture changes invisible. Investigative Ophthalmology and Visual Science (Suppl.), 39, 213.

Palmer, J. (1994) Set-size effects in visual search: The effect of attention is independent of the stimulus for simple tasks. Vision Research, 34, 1703-1721.

Palmer, J. (1995) Attention in visual search: distinguishing four causes of a setsize effect. Current Directions in Psychological Science, 4, 118-123.

Palmer, J., Ames, C.T. and Lindsey, D.T. (1993) Measuring the effect of attention on simple visual search. Journal of Experimental Psychology: Human Perception and Performance, 19, 108-130.

Raney, S.M. and Harvey, L.O. (1995) Visual memory for spatial frequency of Gabor patches. Investigative Ophthalmology and Visual Science, 36, 4, S378.

Regan D, (1985). Storage of spatial frequency information and spatial frequency discrimination. Journal of the Optical Society of America A - Optics and Image Science, 2, 619-621.

Rensink, R.A., O’Regan, J.K. \& Clark, J.J. (1997). To see or not to see: The need for attention to perceive changes in scenes. Psychological Science, 8, 368-373.

Scott-Brown, K.C., Baker, M.R. and Orbach, H.S. (1999) Comparison Blindness. Visual Cognition (special issue).

Scott-Brown, K.C. and Orbach, H.S. (1998) Contrast discrimination, non-uniform patterns and change blindness. Proceedings of the Royal Society, B., 265, 21592166.

Simons, D.J. (1996). In sight, out of mind: when object representations fail. Psychological Science, 7, 301-305. 
Sperling, G. (1960). The information available in brief visual presentations. Psychological Monographs, 72, (whole no. 11).

Wright, M.J. \& Gurney, K.N. (1995) Discrimination of rotation sense in gratings and plaids. Investigative Ophthalmology and Visual Science,(Suppl.) 34, 2911

Wright, M. J.\& Gurney, K. N. (1995). The discrimination of dynamic orientation changes in gratings. Perception, 24, 665-679. 